\title{
Evolution of Endocrine Surgery in India
}

\author{
Sivapatham Vittal
}

Published online: 4 May 2011

(C) Association of Surgeons of India 2011

A Journey of thousand miles begins with a single step. The Endocrine surgery as we see today has grown up to the status of separate subspecialty both nationally and internationally. This is evidenced by the fact that number of academic bodies specially dealing with the Endocrine surgery have been started both in India and Internationally. Today we have IAES (Indian Association of Endocrine Surgeons) a separate section of ASI (Association Surgeons of India), BAETS (British Association of Endocrine and Thyroid Surgeons), Asian Association of Endocrine Surgeons, American Association of Endocrine Surgeons, International Association of Endocrine Surgeons and similar organizations involved in Endocrine Surgery. Separate courses have been started in $\mathrm{MCh}$ in Endocrine Surgery both at Madras Medical College, Chennai and SGPGI (Sanjay Gandhi Post Graduate Institute of Medical Sciences) at Lucknow. The other institutions like CMC (Christian Medical College) Vellore will soon follow in starting MCh in Endocrine Surgery. At a time when the Endocrine Surgery is expanding my thoughts went down memory lane.

While General Surgery is chiefly anatomically oriented, Endocrine Surgery principally aims at restoration of normal physiology, "milieu interior" as first defined by Claude Bernard. There has been a rapid spurt in the diagnosis and evaluation of Endocrine disorders during the last couple of decades mainly due to introduction of Radio Immuno Assay (RIA), ELISA, CLIA (Chemiluminiscence) and other techniques. The availability of these techniques has

\section{S. Vittal $(\bowtie)$}

Emeritus Professor of Surgical Endocrinology,

The Tamil Nadu Dr MGR Medical University, Chennai, India

e-mail: svittal@vsnl.com helped in biochemical diagnosis of Endocrine disorders even before they manifest clinically.

Thus the new concept of biochemical diseases has evolved. It is now possible to operate on a patient even before a particular endocrine gland has show any sign of overt disease or enlargement. For instance in Medullary Carcinoma of Thyroid which is part of MEN Syndrome, we can detect by genetic analysis whether the other family members are likely to be affected, and if so prophylactic therapy in the form of Prophylactic thyroidectomy can be offered.

Accurate localization of tumour has been made possible with Radiological and the other modalities of Imaging like Isotope scan, CT scan, MRI, PET scan, Angiogram and selective venous sampling. Surgical procedures have been made safe by preoperative preparation by appropriate drugs-Pheochromocytoma for example is preoperatively prepared by alpha blockers and if necessary in addition by beta blockers, infusion of crystalloids which help to lower the postoperative morbidity.

On the operative scenario rapid improvement in the surgical techniques has made surgical intervention of endocrine glands possible with little morbidity. Today laparoscopic adrenalectomy has become gold standard if adrenal gland mass is less than $6 \mathrm{~cm}$. As far as other glands are concerned, the place of minimal access surgery is rapidly evolving. The ultrasonic shears may be useful when we are contemplating surgery through minimal access route and also for endoscopic surgery. Likewise in parathyroid surgery, with the advent of better preoperative localization procedures like sestamibi scan and intraoperative localization with rapid intraoperative PTH assay has led to paradigm shift from bilateral cervical approach to targeted or focused parathyroidectomy. Today we have more and more patients of Diabetes Mellitus who are insulin dependent. The right substitution for these patients will be islet cell transplantation. 
With the explosion of growth of biomedical sciences and advances galore in cell based therapy and genetic engineering, solution for Diabetes Mellitus, Obesity and Metabolic diseases may not be very far off.

When I look back at the genesis of Endocrine Surgery in India, it was in 1980, a Division of Endocrine Surgery as an outpatient was started at Government General Hospital, Chennai. I felt the need for a dedicated and independent Department of Endocrine surgery. In the very same year was established a new post of Assistant Professor of Surgical Endocrinology and in 1986 Reader in Surgical Endocrinology was established and I assumed charge as Reader in Surgical Endocrinology. This is actually the first small step towards the commencement of a separate Department of Surgical Endocrinology . It became a full fledged Department in 1987 with the entire necessary infrastructure. Once a dedicated Department was established, management protocols for various Endocrine disorders were instituted. In 1989, another Department of Surgical Endocrinology was started in SGPGI (Sanjay Gandhi Post Graduate Institute of Medical Sciences), Lucknow. It gives me immense satisfaction that I was instrumental in helping the institution to start this department.

As I am writing this, other institutions like CMC (Christian Medical College), Vellore is contemplating to start MCh in Endocrine surgery. I strongly feel Madurai Medical College and Govt. Rajaji Hospital in Madurai is another potential place to start structured training and $\mathrm{MCh}$ in Endocrine surgery. The other places where I feel MCh can be started are AIIMS(All India Institute of Medical Sciences) New Delhi, PGI at Chandigarh and CSM Medical University, Lucknow.

The growth of Endocrine surgery in India is not only measured by starting of subspecialty courses (MCh) in Endocrine Surgery. The Indian Association of Endocrine Surgeons was started as a Section of Association of Surgeons of India in the year 1993. The main aim of this association has been to promote the art and science of Endocrine Surgery in India. It also aims to set standards and training in Endocrine Surgery and to promote the interest in Endocrine Surgery among General Surgeons.

I could wax eloquently for pages of the limitless possibilities in Endocrinology and Endocrine surgery which has opened up new vistas for research. Suffice to say that we as Surgeons of Endocrine systems should incorporate principles of research, documentation and publication of our experience and share our knowledge to each other to alleviate the problems of the suffering humanity for these treatable Endocrine maladies. 